\title{
Descripción morfológica y diferenciación de dos estados de desarrollo de la larva de Oxelytrum lineatocolle (Laporte 1840) (Coleoptera: Silphidae)
}

\section{Morphological description and differentation of two instars of Oxelytrum lineatocolle larvae (Laporte, 1840) (Coleoptera: Silphidae)}

\author{
Edoardo Tosti-Croce ${ }^{1 *}$, Christopher Oses $^{1}$, Alexander OrtlofF$^{2} \&$ Juan Carlos De La Fuente ${ }^{3}$ \\ 1'Departamento de Ciencias Básicas, Facultad de Medicina. Universidad de La Frontera, Avenida Francisco Salazar 01145, \\ Casilla 54-D, Temuco, Chile. \\ 2 Escuela de Medicina Veterinaria, Facultad de Recursos Naturales, Universidad Católica de Temuco, Rudecindo Ortega \\ 02950, Casilla 15-D, Temuco, Chile. \\ ${ }^{3}$ Laboratorio de Criminalística, Policía de Investigaciones de Chile, Avda. Arturo Prat 19, Temuco, Chile. \\ *Email: edoardo.tosticroce@ufrontera.cl.
}

\begin{abstract}
RESUMEN
Se describen morfológicamente dos distintos estados de desarrollo de la larva de Oxelytrum lineatocolle y se reporta por primera vez en cadáveres humanos. Se sugiere que la relación de la longitud de los segmentos de los urogonfios permite identificar estados de desarrollo larval. Además, se describen cuatro caracteres de las "larvas maduras" del género Oxelytrum que permiten diferenciar tres especies presentes en el Neotrópico. La utilidad forense de ésta y otras larvas de coleópteros necrobiontes depende de una correcta identificación, a nivel específico y de estados de desarrollo.
\end{abstract}

Palabras clave: Cadáveres humanos, Estados larvarios, Entomología forense, Neotrópico, Silphinae.

\begin{abstract}
Two different instars of Oxelytrum lineatocolle larva are morphologically described and for the first time reported found in corpses. We suggest using the relationship between length segments of urogomphs to identify instars. In addition, four characters of mature larvae of genre Oxelytrum are described, allowing three species to be differentiated in Neotropical region. The forensic utility of these or other carrion beetle larvae depends on correct identification of species and instars.
\end{abstract}

Keywords: Corpses, Forensic entomology, Instars, Neotropic, Silphinae.

\section{INTRODUCCIÓN}

La familia Silphidae (Superfamilia Staphylinoidea) incluye las subfamilias Nicrophorinae y Silphinae (Lawrence \& Newton 1982). Las especies de esta familia son de hábitos preferentemente necrobiontes y según a la subfamilia que pertenezcan, presentan preferencias de colonización relacionada con el tamaño del cadáver, ya que las especies de la subfamilia Nicrophorinae son conocidas por ser enterradoras de microvertebrados, lo que evita competencia con otros necrófagos, mientras que las especies de la subfamilia Silphinae son capaces de colonizar cadáveres de macromamíferos (Dorsey 1940; Costa et al. 1988; Peck \& Anderson 1985; Peck \& Miller 1993; Watson \& Carlton
2005; Oliva 2012). Los sílfidos se relacionan con los cadáveres dependiendo del estado de desarrollo en que se encuentren, ya que los adultos son depredadores de huevos y larvas de dípteros, además de otras especies necrófilas, mientras que las larvas por lo general presentan hábitos necrófagos (sensu stricto), las que están en estrecha relación con la microsucesión necrobionte (Abbott 1937; Dorsey 1940; Payne \& King 1970; Johnson 1975; Katakura \& Fukuda 1975; Abell et al. 1982), y por lo tanto su presencia ya sea como larva o adulto, podría entregar información importante en una investigación forense.

Las descripciones de las larvas de Silphinae se han realizado principalmente para especies que no son de la región 
neotropical (Böving \& Craighead 1930; Dorsey 1940; Paulian 1952; Watson \& Carlton 2005), mientras que para esta región sólo existen algunas descripciones morfológicas para las larvas maduras de O. erythrurum (Oliva 2004) y $O$. discicolle (Costa et al. 1988) y la determinación de estados de desarrollo de esta última especie por Velázquez \& Viloria (2010). De las nueve especies descritas para el Neotrópico (Oliva 2012), sólo la larva de O. erythrurum ha sido reportada en cadáveres humanos en Argentina (Oliva 2001). Por lo tanto, el objetivo de este trabajo es describir la morfología de las larvas de O. lineatocolle (Laporte 1840) colectadas por primera vez desde cadáveres humanos en Chile, junto con sugerir caracteres morfológicos para la identificación de estados de desarrollo y para diferenciación con las otras dos especies del género Oxelytrum descritas para el Neotrópico.

\section{METODOLOGÍA}

Las larvas en distintos estados de desarrollo fueron recolectadas junto a adultos de $O$. lineacotolle desde cadáveres humanos encontrados en tres sitios de la zona centro sur de Chile: Sector Vegas de Chivilcán (38 42' 41,9” S - 72 35' 28,2” W, Comuna de Temuco, Región de La Araucanía, el 21-11-2006); Sector El Turbio (39 18' 16,6” S - 71 53' 13,3" O, Comuna de Pucón, Región de La Araucanía, el 28-11-2010) y Sector Malhuehue (39²6'
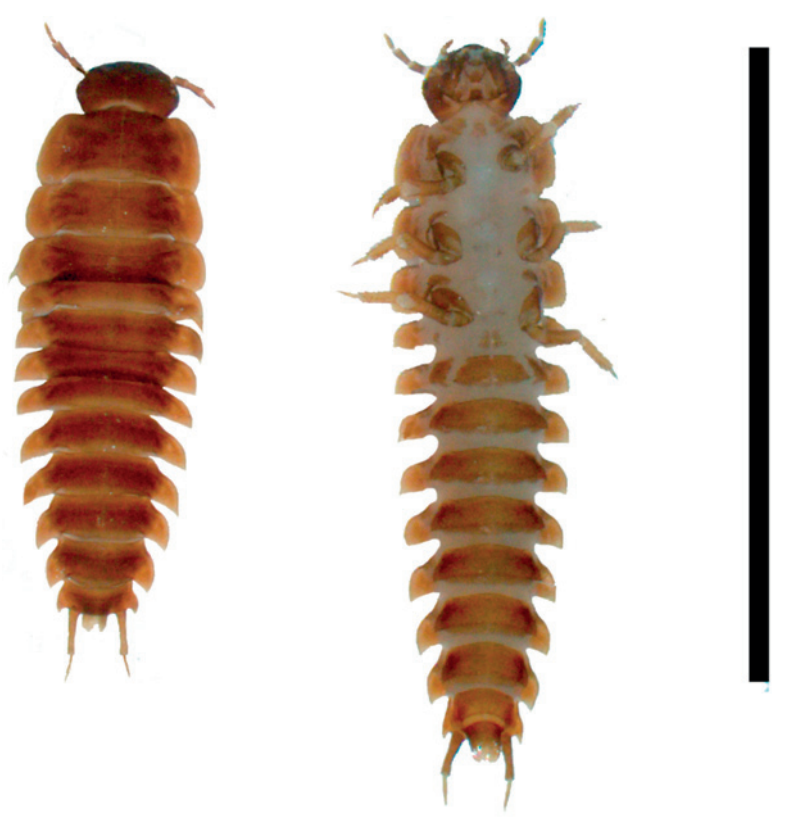

FIGURA 1. Vista dorsal y ventral de la larva inmadura de $O$. lineatocolle. Escala: $10 \mathrm{~mm}$.

Figure 1. Dorsal and ventral view of $O$. lineatocolle inmature larva. Scale: $10 \mathrm{~mm}$.
1,7” S - 72 48’ 20,2” O, Comuna de Lanco, Región de Los Ríos, el 06-04-2012). Las muestras fueron conservadas en solución etanol-acético. El rango de longitud de las larvas fue entre 9 y $24 \mathrm{~mm}$. Se dividieron arbitrariamente las larvas en dos grupos: las que presentaban longitudes inferiores o iguales a $17 \mathrm{~mm}$, las que se consideraron como "larvas inmaduras" (sensu Dorsey 1940) y aquellas con longitudes superiores a los $17 \mathrm{~mm}$, consideradas como "larvas maduras" (sensu Costa et al. 1988; Oliva 2004). Las fotografías ópticas de las larvas se hicieron con una cámara Canon T3i anexada a un estereomicroscopio Olympus SZ-61, mientras que las de microscopía electrónica se obtuvieron a través de un microscopio electrónico de barrido JEOL - JSM-6380LV. Para la descripción morfológica específica, se usaron los caracteres considerados por Dorsey (1940), Costa et al. (1988) y Oliva (2004).

\section{RESULTADOS}

Descripción de la larva (inmadura y madura) de Oxelytrum lineatocolle: Larva campodeiforme, deprimida dorsoventralmente, de color marrón oscuro en las partes más esclerosadas como escudos dorsales, cabeza y patas, mientras que es de color marrón claro en las zonas medianamente esclerosadas como los escudos ventrales y es blanquecina en el resto de la zona ventral (Fig. 1 y 2).
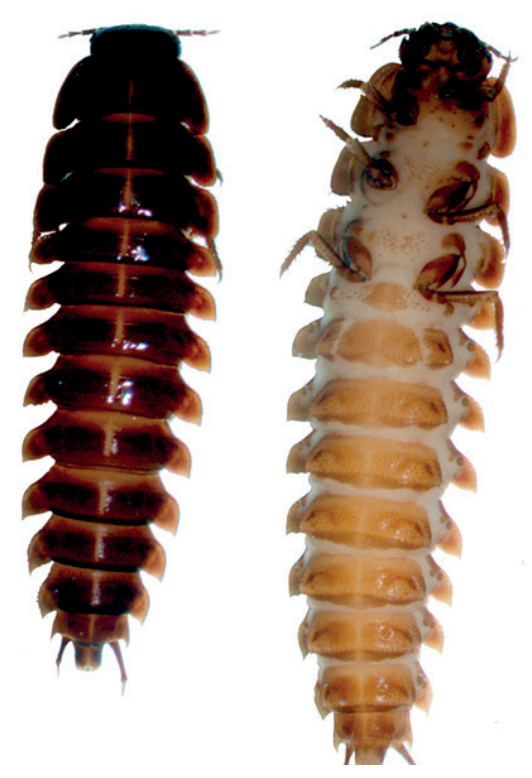

Figura 2. Vista dorsal y ventral de la larva madura de $O$. lineatocolle. Escala: $10 \mathrm{~mm}$.

Figure 2. Dorsal and ventral view of $O$. lineatocolle mature larva. Scale: $10 \mathrm{~mm}$. 
Cabeza proñata más ancha que larga. Antenas de tres segmentos, con los dos primeros más anchos y largos que el apical, el que posee mayor pilosidad que los anteriores (Fig. 3a, 3d). Mandíbulas simétricas y curvas con dos procesos distales, ambos con bordes internos dentados, sin área molar (Fig. 3b, 3e). Maxila con cuatro setas simples, ubicadas en la zona latero inferior media de la gálea; en la zona ventral de la gálea a la altura de la base del palpo maxilar; en la zona latero inferior del estipe y en la zona ventral media del tercio proximal del estipe (Fig. 3c, 3f). Palpo maxilar de tres segmentos, donde los dos proximales poseen una longitud similar y se ensanchan hacia el ápice, mientras que el segmento apical es más corto y posee forma ahusada con base truncada (Fig. 4a, 4d). Palpo labial con dos segmentos, donde el proximal es aproximadamente el doble de largo del segmento distal, el que además es de menor diámetro y ápice truncado que posee estructuras circulares solevantadas, posiblemente sensoriales (Fig. 4b, 4e). Labio con lígula bilobulada, con área ventral granulada, mientras que el área dorsal está cubierta por una serie de penachos formados por 3 a 6 procesos filiformes (Fig. 4c, 4f). Gálea con pilosidad lisa. Lacinia de 11 dientes.

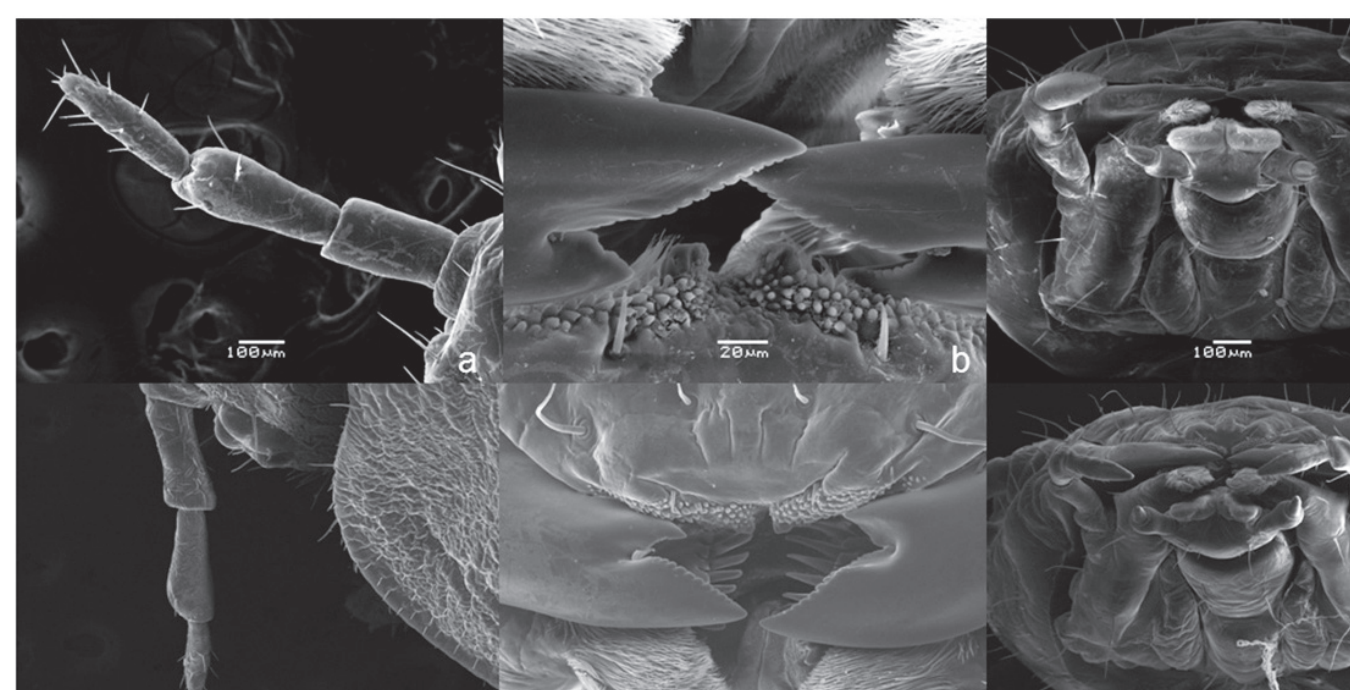

Figura 3. Antena, mandíbulas y vista ventral de la cabeza de la larva inmadura (a, b, c respectivamente) y madura (d, e, f respectivamente) de $O$. lineatocolle.

Figure 3. Antenna, mandibles and head (ventral view) of immature (a, b, c respectively) and mature (d, e, f respectively) larva of $O$. lineatocolle.

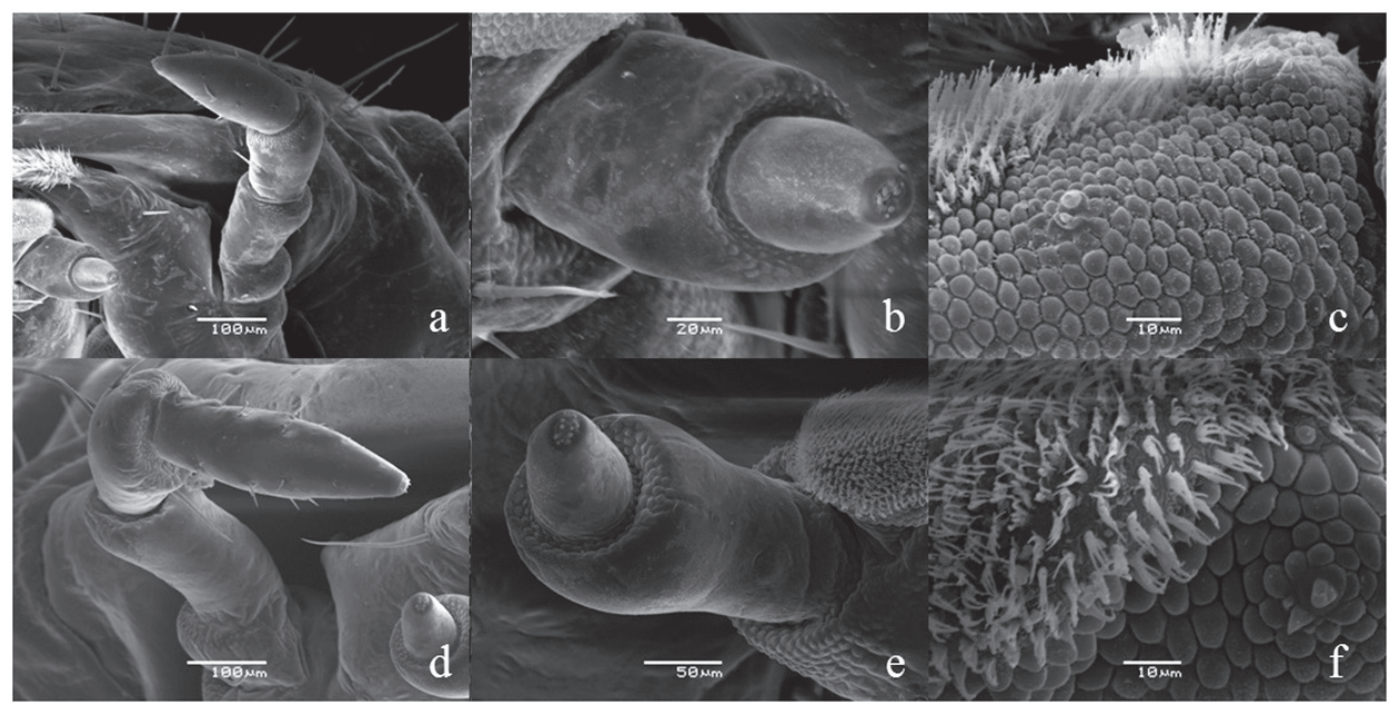

Figura 4. Palpo maxilar, labial y lígula de la larva inmadura (a, b, c respectivamente) y madura (d, e, f respectivamente) de $O$. lineatocolle.

Figure 4. Maxillary, labial palpiger and ligula of immature (a, b, c respectively) and mature (d, e, f respectively) larva of $O$. lineatocolle. 
Escudos dorsales con procesos laterales laminares planos de bordes aserrados, con pilosidad de longitud irregular, los que apuntan generalmente en sentido diagonal-posterior al eje longitudinal de la larva, con una seta más larga que las demás en el ápice (Fig. 5a, 5b, 5d, 5e). Los escudos ventrales son más cortos y angostos que los dorsales. Los escudos ventrales abdominales presentan esclerotización completa sólo a partir del tercer segmento y tienen forma de trapecio, con bordes irregulares sin procesos laterales. El segundo escudo es discontinuo, con dos áreas pequeñas laterales y claramente separadas de la central, mientras que el segundo presenta dos cortes que dejan tres áreas esclerosadas, la central aproximadamente cuatro veces más ancha que las dos laterales.

Urogonfios de dos segmentos: el primero subcilíndrico y el segundo subulado. En las larvas inmaduras, el primer segmento es aproximadamente dos veces el segundo (Fig. 1, $5 c)$, mientras que en las larvas maduras es aproximadamente tres veces su largo (Fig. 2, 5f).

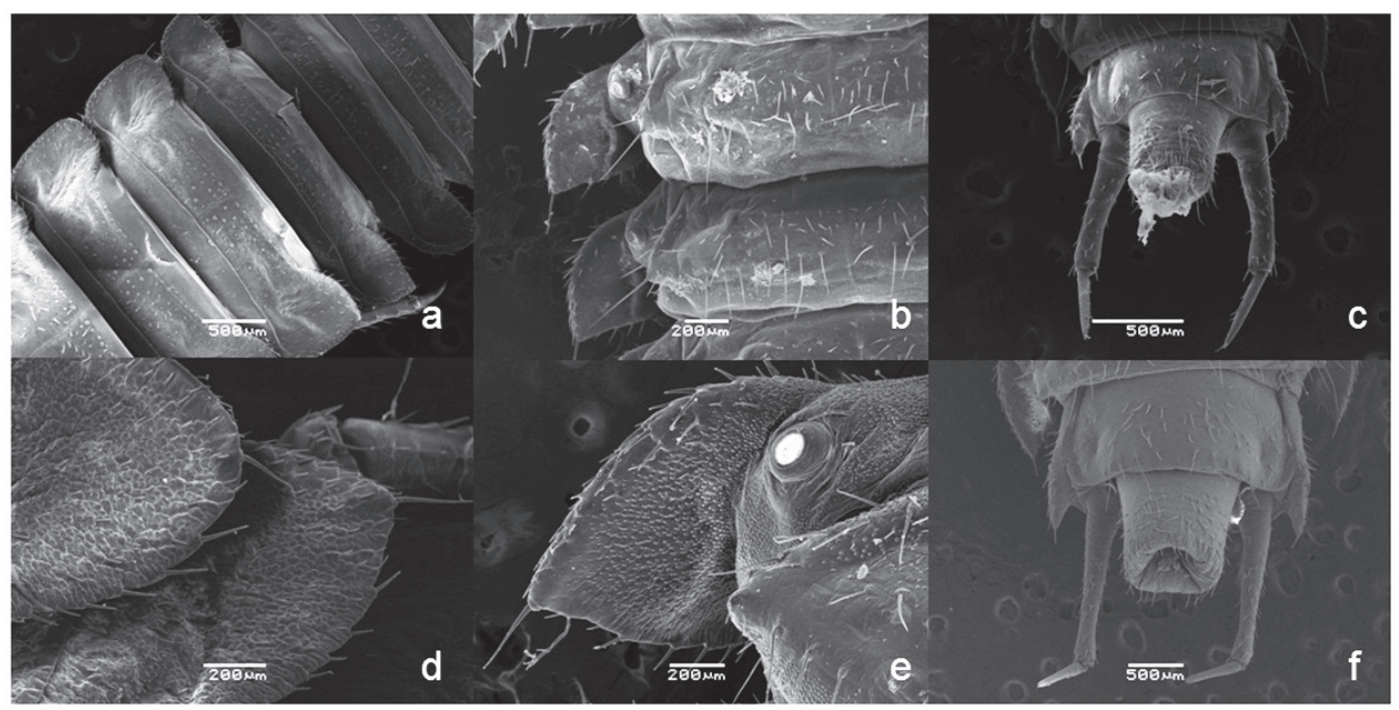

FiguRa 5. Vista dorsal y ventral de los escudos torácicos y abdominales, y urogonfios de la larva inmadura (a, b, c respectivamente) y madura (d, e, f respectivamente) de $O$. lineatocolle.

FiguRE 5. Dorsal and ventral view of thoracic and abdominal shields, and urogomphs of immature (a, b, c respectively) and mature (d, e, $\mathrm{f}$ respectively) larva of $O$. lineatocolle.

\section{DISCUSIÓN}

Se registra por primera vez la presencia de larvas y adultos de $O$. lineatocolle en cadáveres humanos en Chile, ya que sólo un adulto de esta especie había sido colectada desde un cuerpo en Neuquén, Argentina (Oliva \& Di Iorio 2008). Al comparar ambos estados de desarrollo de la larva de esta especie, se observa una diferencia en la relación entre la longitud de los segmentos de los urogonfios, la cual sólo se había considerado anteriormente como carácter para diferenciar especies (Dorsey 1940). Debido a que para este trabajo se contó con larvas de $O$. lineatocolle de distintos tamaños, se pudo observar que este cambio en la relación de los segmentos de los urogonfios también permite diferenciar, al menos dos estados diferentes de desarrollo, que denotan tiempos distintos de colonización de un cuerpo por esta especie. Por este motivo, se sugiere tener precaución en la utilización de este carácter sólo para identificar especies. Otras publicaciones también han propuesto mediciones específicas entre otras estructuras de los distintos estados de desarrollo larvarios, con el fin de determinar la cantidad de estados en varias especies de sílfidos (Watson \& Carlton 2005; Velásquez \& Viloria 2010).

Al comparar las descripciones de las larvas maduras de $O$. lineatocolle con las de $O$. discicolle y $O$. erythrurum (Costa et al. 1988; Oliva 2004) se pudo establecer cuatro características claves para diferenciarlas a nivel específico (Tabla 1), lo que no significa que éstos sean exclusivos de estas especies. Debido a que existe desconocimiento de las otras seis especies del género Oxelytrum descritas para la Región neotropical, la correcta identificación específica de estos insectos necrobiontes es fundamental para poderlos considerar como prueba forense. 
TABLA 1: Diferencias morfológicas de las larvas maduras de tres especies de Oxelytrum.

Table 1: Morphological differences of mature larvae of three Oxelytrum species.

\begin{tabular}{lccc}
\hline CARÁCTER MORFOLÓGICO & $\begin{array}{c}\text { O. discicolle } \\
\text { (Costa et al. 1988) }\end{array}$ & $\begin{array}{c}\text { O. erythrurum } \\
\text { (Oliva 2004) }\end{array}$ & $\begin{array}{c}\text { O. lineatocolle } \\
\text { (presente trabajo) }\end{array}$ \\
\hline Setas en maxila & 5 & 3 & 4 \\
Mandíbulas & Simétricas & Asimétricas & Simétricas \\
Cono sensorial & Presente & Ausente & Ausente \\
Relación segmento proximal: distal de & $4: 1$ & $2: 1$ & $3: 1$ \\
los urogonfios & & & \\
\hline
\end{tabular}

\section{AGRADECIMIENTOS}

Se agradece a CONICYT por el financiamiento al proyecto FONDEF D09I1035, a la Policía de Investigaciones de Chile (PDI), al Ministerio Público, y en especial al Sr. Hugo Pacheco, técnico del Laboratorio de Microscopía Electrónica de la UdeC (Q.E.P.D.).

\section{BIBLIOGRAFÍA}

Аввотт, C.E. 1937. The necrophilous habit in Coleoptera. Bulletin in the Brooklyn Entomological Society 32:202-204.

Böving, A.G. \& Craighead, F.C.1930. An illustrated synopsis of the principal larval forms of the orden Coleoptera. Entomologica Americana N.S (11), New York. 351 pp.

Dorsey, C.K. 1940. A comparative study of the larvae of six species of Silpha. Annuals Entomological Society of America 33(1):120-139.

Costa, C. Vanin, S.A. \&CAsari-Chen, S.A. 1988. Larvas de Coleoptera do Brasil. Museu de Zoologia, Universida de de São Paulo, São Paulo.

Johnson, M.D. 1975. Seasonal and microseral variations in the insect populations on carrion. The American Midland Naturalist 93(1):79-99.

KataKudA, H. \& FuKUdA, H. 1975. Faunal makeup of ground and carrion beetles in Kamiotoineppu, Hokkaido University Nakagawa Experiment Forest, Northern Japan, with some notes of related problems. Research Bulletins of the College Experiment Forests 32 (1):75-92.
LaWrence, J.F. \& Newton, A.F. 1982. Evolution and classification of beetles. Ann. Rev. Ecol. Syst. 13:261-90.

Oliva, A. 2004. Description of the larva of Oxelytrum erythrurum (Blanchard, 1849) (Coleoptera, Silphidae). Bulletin S.R.B.E./K.B.V.E. 140:141-144.

Oliva, A. 2012. A new species of Oxelytrum Gistel (Coleoptera, Silphidae) from Southern Argentina, with a key to the species of the genus. Zookeys 203:1-14.

Oliva, A. \& Di Iorio, O. 2008. Silphidae. En: Biodiversidad de artrópodos argentinos, vol. 2 (Eds. Claps, L.E., Debandi, G. \& S. Roig-Juñent), pp. 461-470. Sociedad Entomológica Argentina, Tucumán.

Paulian, R. 1952. Premiers États des Staphylinoidea (Coloeptera). Thèses de Doctéur. Université de Paris. Paris, France.

Payne, J.A. \& King, E.W.1970. Coleoptera associated with pig carrion. Entomologist Monthly Magazine 105:224-232.

PeCK, S.B. \& Anderson, R.S. 1985. Taxonomy, phylogeny and biogeography of the carrion beetles of Latin America (Coleoptera: Silphidae). Quaestiones Entomologicae 21:247-317.

Peck, S.B. \& Miller, S.E. 1993. A catalog of the Coleoptera of America North of Mexico, Family: Silphidae. United States Department of Agriculture: 47-81.

Velásquez, Y. \&Viloria, A.L. 2010. Instar determination of the Neotropical Beetle Oxelytrum discicolle (Coleoptera: Silphidae). Journal of Medical Entomology 47(5):723726.

Watson, E.J. \& CARLton, C.E. 2005. Succession of forensically significant carrion beetle larvae on large carcasses (Coleoptera: Silphidae). Southeastern Naturalist 4(2):335345 .

Recibido: 08.01.14

Aceptado: 28.08.14 